\title{
Unitary realizations of the ideal phase measurement
}

\author{
F. Buscemi, G. M. D'Ariano, and M. F. Sacchi \\ Quantum Optics $\&$ Information Group \\ Università degli Studi di Pavia and INFM Unità di Pavia \\ via A. Bassi 6, I-27100 Pavia, Italy
}

\begin{abstract}
We explicitly construct a large class of unitary transformations that allow to perform the ideal estimation of the phase-shift on a single-mode radiation field. The ideal phase distribution is obtained by heterodyne detection on two radiation modes after the interaction.
\end{abstract}

The quantum estimation of an unknown phase shift - the so called quantum phase measurement - is the essential problem of high sensitive interferometry, and has received much attention in quantum optics [1]. For a single-mode electromagnetic field, the measurement cannot be achieved exactly, even in principle, due to the lack of a unique self-adjoint operator [2]. In fact, the absence of a proper self-adjoint operator is mainly due to the semi-boundedness of the spectrum of the number operator $[3,4]$, which is canonically conjugated to the phase in the sense of a Fourier-transform pair [5].

This observation opened the route for an exact phase measurement in terms of two-mode fields, where a phase-difference operator becomes conjugated to an unbounded number-difference operator [6]. In fact, a concrete experimental setup using unconventional heterodyne detection has been suggested [7] for this kind of measurement. However in the single-mode case, no feasible scheme that can provide the optimal phase measurement has been devised yet.

The most general and concrete approach to the problem of the phase measurement is quantum estimation theory [8], a framework that has become popular only in the last ten years in the field of quantum information. Quantum estimation theory provides a more general description of quantum statistics in terms of POVM's (positive operator-valued measures) and gives the theoretical definition of an optimized phase measurement. The most powerful method for deriving the optimal phase measurement was given by Holevo [9] in the 
covariant case. In this way the optimal POVM for phase estimation has been derived for a single-mode field. More generally, the problem of estimating the phase shift has been addressed in Ref. [10] for any degenerate shift operator with discrete spectrum, either bounded, bounded from below, or unbounded, extending the Holevo method for the covariant estimation problem.

As already stated, quantum estimation theory provides the optimal POVM for the phase measurement. This writes in terms of projectors on SusskindGlogower states [11]

$$
d \mu(\varphi)=\frac{d \varphi}{2 \pi}\left|e^{i \varphi}\right\rangle\left\langle e^{i \varphi}\right|
$$

where $\left|e^{i \varphi}\right\rangle=\sum_{n=0}^{\infty} e^{i \varphi n}|n\rangle$. Notice that the states $\left|e^{i \varphi}\right\rangle$ are not normalizable, neither orthogonal, however they provide a resolution of the identity, and thus guarantee the completeness of the POVM, namely

$$
\int_{0}^{2 \pi} d \mu(\varphi)=I
$$

For a system in state $\rho$, the POVM in Eq. (1) gives the ideal phase distribution $p(\varphi)$ according to Born's rule

$$
p(\varphi)=\operatorname{Tr}[d \mu(\varphi) \rho]=\frac{d \varphi}{2 \pi}\left\langle e^{i \varphi}|\rho| e^{i \varphi}\right\rangle
$$

In this Letter we will explicitly construct some unitary transformations that allows to perform the ideal phase measurement described by the POVM in Eq. (1). First, we will introduce an isometry $\tilde{V}$ which enlarges the Hilbert space of the system ( say $\mathcal{H}_{a}$ for mode $a$ ) to the tensor product $\mathcal{H}_{a} \otimes \mathcal{H}_{b}$ for two modes $a$ and $b$. Then, we will prove that the exact measurement of the complex photocurrent $Z=a-b^{\dagger}$ provides through its marginal distribution the ideal probability density $p(\varphi)$ of Eq. (3). Finally, we will construct a large class of unitary operators on $\mathcal{H}_{a} \otimes \mathcal{H}_{b} \otimes \mathcal{H}_{c}$, where $\mathcal{H}_{c}$ denotes the Hilbert space of an ancillary arbitrary system, such that the isometry $\tilde{V}$ is realized with unit probability.

We start by introducing the eigenstates of the heterodyne photocurrent $Z=$ $a-b^{\dagger}[12,13,7]$

$$
\left.Z|D(z)\rangle\rangle_{a b}=z|D(z)\rangle\right\rangle_{a b}
$$

where $D(z)=\exp \left(z a^{\dagger}-z^{*} a\right)$ denotes the displacement operator. Here and in the following we use the notation [14] for bipartite pure states on $\mathcal{H}_{a} \otimes \mathcal{H}_{b}$ 


$$
\left.\left.|A\rangle\rangle_{a b}=\sum_{n, m=0}^{\infty} A_{n m}|n\rangle_{a} \otimes|m\rangle_{b} \equiv A \otimes I_{b}|I\rangle\right\rangle_{a b} \equiv I_{a} \otimes A^{\tau}|I\rangle\right\rangle_{a b},
$$

where $A^{\tau}$ denotes the transposed operator with respect to some pre-chosen orthonormal basis. The states $|D(z)\rangle\rangle_{a b}$ are orthogonal in Dirac sense over the complex plane, namely

$$
{ }_{a b}\langle\langle D(\alpha) \mid D(\beta)\rangle\rangle_{a b}=\pi \delta^{(2)}(\alpha-\beta) \equiv \pi \delta(\operatorname{Re} \alpha-\operatorname{Re} \beta) \delta(\operatorname{Im} \alpha-\operatorname{Im} \beta) .(6)
$$

They also provides a basis for $\mathcal{H}_{a} \otimes \mathcal{H}_{b}$ as follows

$$
\left.\int_{\mathbb{C}} \frac{d^{2} z}{\pi}|D(z)\rangle\right\rangle_{a b a b}\left\langle\langle D(z)|=I_{a} \otimes I_{b} .\right.
$$

The measurement of the complex photocurrent $Z$ can be performed through unconventional heterodyne detection [13] with both the signal $a$ and the imageband $b$ non-vacuum (in usual heterodyne detection the image-band mode is in the vacuum, thus providing the well-known coherent-state POVM). The measurement of $Z$ is also equivalent to two separate homodyne measurements on modes $\frac{1}{\sqrt{2}}(b \pm a)$. In fact, consider the $50 / 50$ beam splitter operator $R=$ $\exp \left[\frac{\pi}{4}\left(a^{\dagger} b-a b^{\dagger}\right)\right]$ that realizes the unitary transformation

$$
R\left(\begin{array}{l}
a \\
b
\end{array}\right) R^{\dagger}=\frac{1}{\sqrt{2}}\left(\begin{array}{rr}
1 & -1 \\
1 & 1
\end{array}\right)\left(\begin{array}{l}
a \\
b
\end{array}\right) .
$$

Upon denoting with $|x\rangle_{a}$ and $|y\rangle_{b}$ the eigenstates of the quadratures $X_{a}=$ $\left(a+a^{\dagger}\right) / \sqrt{2}$ and $Y_{b}=\left(i b^{\dagger}-i b\right) / \sqrt{2}$, one has the following identity [15]

$$
\left.R\left(|x\rangle_{a a}\langle x|\otimes| y\rangle_{b b}\langle y|\right) R^{\dagger}=|D(x+i y)\rangle\right\rangle_{a b a b}\langle\langle D(x+i y)| .
$$

Notice also that this kind of measurement is performed in the teleportation protocol for continuous variable of Braunstein-Kimble scheme [16,17].

We can now write the isometry $\tilde{V}$ such that the transformation

$$
T(\rho)=\tilde{V} \rho \tilde{V}^{\dagger}
$$

maps the state of the system $\rho \in \mathcal{H}_{a}$ to a two-mode state in $\mathcal{H}_{a} \otimes \mathcal{H}_{b}$. The operator $\tilde{V}$ has the form

$$
\left.\tilde{V}=\frac{1}{\sqrt{2 \pi}} \int_{\mathbb{C}} d^{2} \alpha f(|\alpha|)|D(\alpha)\rangle\right\rangle_{a b a}\left\langle e^{i \arg \alpha}\right| .
$$


By choosing $f(t)$ as an arbitrary function satisfying the condition

$$
\int_{0}^{+\infty} d t t|f(t)|^{2}=\frac{1}{\pi}
$$

it follows that $\tilde{V}$ is an isometry, namely $\tilde{V}^{\dagger} \tilde{V}=I_{a}$.

It is easy to check that the transformation (10) has the following covariance symmetry

$$
T\left(e^{i \theta a^{\dagger} a} \rho e^{-i \theta a^{\dagger} a}\right)=e^{i \theta a^{\dagger} a} \otimes e^{-i \theta b^{\dagger} b} T(\rho) e^{-i \theta a^{\dagger} a} \otimes e^{i \theta b^{\dagger} b} .
$$

We can now evaluate the probability density of getting outcome $z \in \mathbb{C}$ through the measurement of the photocurrent $Z$. One has

$$
\begin{aligned}
p(z) & =\frac{1}{\pi} \operatorname{Tr}\left[\tilde{V} \rho \tilde{V}^{\dagger}|D(z)\rangle\right\rangle_{a b a b}\langle\langle D(z)|] \\
& =\frac{1}{2}|f(|z|)|^{2}{ }_{a}\left\langle e^{i \varphi}|\rho| e^{i \varphi}\right\rangle_{a}, \quad \varphi=\arg z
\end{aligned}
$$

From condition in Eq. (12), it follows that the marginal distribution on the statistical variable $\varphi=\arg z$ corresponds to the ideal distribution of the phase for any input state $\rho$.

In the following we explicitly construct some unitary realizations of the map in Eq. (10). We start by defining the operators in $\mathcal{L}\left(\mathcal{H}_{a} \otimes \mathcal{H}_{b}\right)$

$$
V=\tilde{V}\left(I_{a} \otimes_{b}\langle\chi|\right), \quad V^{\dagger}=\left(I_{a} \otimes|\chi\rangle_{b}\right) \tilde{V}^{\dagger}
$$

where $|\chi\rangle$ is an arbitrary normalized state in $\mathcal{H}_{b}$, and the tensor notation $I_{a} \otimes_{b}\langle\chi|$ represents a linear operator from $\mathcal{H}_{a} \otimes \mathcal{H}_{b}$ to $\mathcal{H}_{a}$ (the bra ${ }_{b}\langle\chi|$ can be regarded as a linear functional from $\mathcal{H}_{b}$ to $\mathbb{C}$ ). Similarly, $I_{a} \otimes|\chi\rangle_{b}$ represents an operator from $\mathcal{H}_{a}$ to $\mathcal{H}_{a} \otimes \mathcal{H}_{b}$. Notice that both $V V^{\dagger}$ and $V^{\dagger} V$ are projectors, namely

$$
V V^{\dagger} V V^{\dagger}=V V^{\dagger}, \quad V^{\dagger} V V^{\dagger} V=V^{\dagger} V
$$

Upon introducing an arbitrary Hilbert space $\mathcal{H}_{c}$ (also finite dimensional), we construct the following operator

$$
U=V \otimes W W^{\dagger}-V^{\dagger} \otimes W^{\dagger} W+\left(I-V^{\dagger} V\right) \otimes W^{\dagger}+\left(I-V V^{\dagger}\right) \otimes W
$$

where $W$ is a linear operator in $\mathcal{H}_{c}$. Under the conditions 


$$
W^{2}=\left(W^{\dagger}\right)^{2}=0, \quad W W^{\dagger}+W^{\dagger} W=I_{c},
$$

one can easily check that $W W^{\dagger}$ and $W^{\dagger} W$ are projectors orthogonal each other, and $U$ is unitary. Consider now the transformation of the system prepared in a state $\rho \otimes \sigma \otimes \mu$ which has been evolved through the unitary $U$ and traced over the ancillary space $\mathcal{H}_{c}$. One has

$$
\begin{aligned}
& \operatorname{Tr}_{c}\left[U(\rho \otimes \sigma \otimes \mu) U^{\dagger}\right]=V(\rho \otimes \sigma) V^{\dagger} \operatorname{Tr}\left[W W^{\dagger} \mu\right] \\
& +V(\rho \otimes \sigma)\left(I-V V^{\dagger}\right) \operatorname{Tr}\left[W^{\dagger} \mu\right]+V^{\dagger}(\rho \otimes \sigma) V \operatorname{Tr}\left[W^{\dagger} W \mu\right] \\
& -V^{\dagger}(\rho \otimes \sigma)\left(I-V^{\dagger} V\right) \operatorname{Tr}[W \mu]-\left(I-V^{\dagger} V\right)(\rho \otimes \sigma) V \operatorname{Tr}\left[W^{\dagger} \mu\right] \\
& +\left(I-V^{\dagger} V\right)(\rho \otimes \sigma)\left(I-V^{\dagger} V\right) \operatorname{Tr}\left[W W^{\dagger} \mu\right]+\left(I-V V^{\dagger}\right)(\rho \otimes \sigma) V^{\dagger} \operatorname{Tr}[W \mu] \\
& +\left(I-V V^{\dagger}\right)(\rho \otimes \sigma)\left(I-V V^{\dagger}\right) \operatorname{Tr}\left[W^{\dagger} W \mu\right] .
\end{aligned}
$$

The map in Eq. (10) can then be achieved by the unitary transformation $U$, by taking

$$
\sigma=|\chi\rangle_{b b}\langle\chi|
$$

and $\mu$ such that

$$
\operatorname{Tr}\left[W W^{\dagger} \mu\right]=1, \quad \operatorname{Tr}[W \mu]=\operatorname{Tr}\left[W^{\dagger} \mu\right]=0
$$

We summarize the conditions on the measurement scheme: $f(t),|\chi\rangle, W, \mu$ in Eqs. (11), (15), (17), (19) are arbitrary, provided that conditions (12), (18), (21) are satisfied.

As an example, consider the case in which the space $\mathcal{H}_{c}$ pertains to a radiation mode $c$. One can take

$$
W=\sum_{n=0}^{\infty}|2 n\rangle_{c c}\langle 2 n+1|, \quad \mu=| 0\rangle_{c c}\langle 0|,
$$

thus obtaining

$$
T(\rho)=\operatorname{Tr}_{c}\left[U\left(\rho \otimes|\chi\rangle_{b b}\langle\chi|\otimes| 0\rangle_{c c}\langle 0|\right) U^{\dagger}\right]
$$

We notice that the "pseudo-spin" operator $W$ in Eq. (22) has been introduced also in Refs. $[18,19]$ in the context of Bell's inequalities for continuous variable.

The result in Eq. (23) is similarly obtained for a qubit system in $\mathcal{H}_{c}$, with $W=|0\rangle_{c c}\langle 1|$. 
In conclusion, a large class of unitary realizations of the ideal phase measurement of a single-mode radiation field has been presented. These unitary evolutions act on the Hilbert space $\mathcal{H}_{a} \otimes \mathcal{H}_{b} \otimes \mathcal{H}_{c}$, where $\mathcal{H}_{a}$ and $\mathcal{H}_{b}$ are referred to radiation modes, and $\mathcal{H}_{c}$ pertains to an arbitrary system. By suitably preparing the state of the systems in $\mathcal{H}_{b}$ and $\mathcal{H}_{c}$, the ideal phase distribution for the input state $\rho \in \mathcal{H}_{a}$ is obtained through heterodyne detection performed after the interaction on modes $a$ and $b$.

\section{Acknowledgments}

This work has been sponsored by INFM through the project PRA-2002CLON, and by EEC through the ATESIT project IST-2000-29681.

\section{References}

[1] Physica Scripta T 48 (1993) (special issue on Quantum Phase and Phase Dependent measurements).

[2] F. London, Z. Phys. 37, 915 (1926); 40, 193 (1927).

[3] J. H. Shapiro and S. R. Shepard, Phys. Rev. A 43, 3795 (1991).

[4] M. Ban, Phys. Rev. A 50, 2785 (1994).

[5] J. H. Shapiro, Physica Scripta T 48, 105 (1993).

[6] A. Luis and L. L. Sánches-Soto, Phys. Rev. A 48, 4702 (1993).

[7] G. M. D'Ariano and M. F. Sacchi, Phys. Rev. A 52, R4309 (1995).

[8] C. W. Helstrom, Quantum Detection and Estimation Theory, Academic, New York, 1976.

[9] A. S. Holevo. Probabilistic and statistical aspects of quantum theory, NorthHolland, Amsterdam, 1982.

[10] G. M. D'Ariano, C. Macchiavello, and M. F. Sacchi, Phys. Lett. A 248, 103 (1998).

[11] L. Susskind and J. Glogower, Physics 1, 49 (1964).

[12] H. P. Yuen and J. H. Shapiro, IEEE Trans. Inform. Theory IT 26, 78 (1980).

[13] J. H. Shapiro and S. S. Wagner, IEEE J. Quantum Electron. QE 20, 803 (1984).

[14] G. M. D'Ariano, P. Lo Presti, and M. F. Sacchi, Phys. Lett. A 272, 32 (2000).

[15] G. M. D’Ariano and M. F. Sacchi, Mod. Phys. Lett. B. 11, 1263 (1997). 
[16] S. L. Braunstein and H. J. Kimble, Phys. Rev. Lett. 80, 869 (1998).

[17] S. L. Braunstein, G. M. D'Ariano, G. J. Milburn, and M. F. Sacchi, Phys. Rev. Lett. 84, 3486 (2000).

[18] Z.-B. Chen, J.-W. Pan, G. Hou, and Y.-D. Zhang, Phys. Rev. Lett. 88, 040406 (2003).

[19] J. Larsson, Phys. Rev. A 67, 022108 (2003). 